Research Article

\title{
Applicability of Dmax Method on Heart Rate Variability to Estimate the Lactate Thresholds in Male Runners
}

\author{
Eduardo Marcel Fernandes Nascimento (D, Diego Antunes, \\ Paulo Cesar do Nascimento Salvador, Fernando Klitzke Borszcz, \\ and Ricardo Dantas de Lucas
}

\begin{abstract}
Physical Effort Laboratory, Sports Center, Federal University of Santa Catarina, Florianopolis, Brazil
Correspondence should be addressed to Eduardo Marcel Fernandes Nascimento; eduardomarcel@usp.br
\end{abstract}

Received 1 April 2019; Revised 20 June 2019; Accepted 3 July 2019; Published 19 September 2019

Academic Editor: Mark Willems

Copyright (c) 2019 Eduardo Marcel Fernandes Nascimento et al. This is an open access article distributed under the Creative Commons Attribution License, which permits unrestricted use, distribution, and reproduction in any medium, provided the original work is properly cited.

\begin{abstract}
Introduction. The purpose of this study was to evaluate the application of the Dmax method on heart rate variability (HRV) to estimate the lactate thresholds (LT), during a maximal incremental running test (MIRT). Methods. Nineteen male runners performed two MIRTs, with the initial speed at $8 \mathrm{~km} \cdot \mathrm{h}^{-1}$ and increments of $1 \mathrm{~km} \cdot \mathrm{h}^{-1}$ every 3 minutes, until exhaustion. Measures of HRV and blood lactate concentrations were obtained, and lactate $\left(\mathrm{LT}_{1}\right.$ and $\left.\mathrm{LT}_{2}\right)$ and HRV (HRVT $\mathrm{DMAX1}_{1}$ and HRVT $\left._{\text {DMAX2 }}\right)$ thresholds were identified. ANOVA with Scheffe's post hoc test, effect sizes $(d)$, the bias $\pm 95 \%$ limits of agreement (LoA), standard error of the estimate (SEE), Pearson's ( $r$ ), and intraclass correlation coefficient (ICC) were calculated to assess validity. Results. No significant differences were observed between $\mathrm{HRVT}_{\mathrm{DMAX}}$ and $\mathrm{LT}_{1}$ when expressed for speed $\left(12.1 \pm 1.4 \mathrm{~km} \cdot \mathrm{h}^{-1}\right.$ and $11.2 \pm 2.1 \mathrm{~km} \cdot \mathrm{h}^{-1} ; p=0.55 ; d=0.45 ; r=0.46 ;$ bias $\pm \mathrm{LoA}=0.8 \pm 3.7 \mathrm{~km} \cdot \mathrm{h}^{-1} ; \mathrm{SEE}=1.2 \mathrm{~km} \cdot \mathrm{h}^{-1}(95 \% \mathrm{CI}$, 0.9-1.9)). Significant differences were observed between $\mathrm{HRVT}_{\mathrm{DMAX} 2}$ and $\mathrm{LT}_{2}$ when expressed for speed $\left(12.0 \pm 1.2 \mathrm{~km} \cdot \mathrm{h}^{-1}\right.$ and $14.1 \pm 2.5 \mathrm{~km} \cdot \mathrm{h}^{-1} ; p=0.00 ; d=1.21 ; r=0.48$; bias $\left.\pm \mathrm{LoA}=-1.0 \pm 1.8 \mathrm{~km} \cdot \mathrm{h}^{-1} ; \mathrm{SEE}=1.1 \mathrm{~km} \cdot \mathrm{h}^{-1}(95 \% \mathrm{CI}, 0.8-1.6)\right)$, respectively. Reproducibility values were found for the $\mathrm{LT}_{1} \quad\left(\mathrm{ICC}=0.90 ;\right.$ bias $\left.\pm \mathrm{LoA}=-0.7 \pm 2.0 \mathrm{~km} \cdot \mathrm{h}^{-1}\right), \quad \mathrm{LT}_{2} \quad\left(\mathrm{ICC}_{\mathrm{C}}=0.97\right.$; bias $\left.\pm \mathrm{LoA}=-0.1 \pm 1.1 \mathrm{~km} \cdot \mathrm{h}^{-1}\right), \mathrm{HRVT}_{\mathrm{DMAX} 1} \quad\left(\mathrm{ICC}=0.48 ; \quad\right.$ bias $\left.\pm \mathrm{LoA}=-0.2 \pm 3.4 \mathrm{~km} \cdot \mathrm{h}^{-1}\right)$, and $\mathrm{HRVT}_{\mathrm{DMAX} 2} \quad(\mathrm{ICC}=0.30$; bias $\left.\pm \mathrm{LoA}=0.3 \pm 3.5 \mathrm{~km} \cdot \mathrm{h}^{-1}\right)$. Conclusions. The Dmax method applied over a HRV dataset allowed the identification of $\mathrm{LT}_{1}$ that is close to aerobic threshold, during a MIRT.
\end{abstract}

\section{Introduction}

The autonomic cardiac drive can be investigated by the heart rate variability (HRV), which is characterized as a variation quantified in milliseconds between RR intervals [1]. A predominance of parasympathetic nervous system (PNS) activity is observed at rest and low effort intensities. In approximately $50-60 \%$ of the maximum oxygen uptake $\left(\mathrm{VO}_{2 \mathrm{MAX}}\right)$, a significant vagal withdrawal occurs [2]. The aerobic threshold $(\mathrm{AeT})$ has been related to that intensity [3-5], i.e., the exercise intensity which lactate concentrations [La] initiate to increase beyond resting values and are frequently called "lactate threshold" (LT) [6, 7]. On the other hand, in intensities above the AeT, there is a gradual and constant increase in activation in the sympathetic nervous system (SNS), and a marked increase in the physiological responses related to the anaerobic threshold (AnT) can be observed $[3,4]$. That intensity is corresponding to maximal lactate steady state (MLSS), i.e., the highest constant exercise intensity output that can be maintained over time without continual [La] accumulation $[3,6]$.

Since AeT and AnT are good indexes of ideal training intensity and determinants of endurance performance $[3,4,6]$, there is an obvious and growing interest in proposing different methods to estimate that intensities, mainly in relation to MSSL, considered gold standard endurance performance marker $[3,6,8]$. Among the different methods, the HRV thresholds (HRVTs) have been highlighted 
[5, 9-15]. The HRVT method is accessible and of low cost to use compared to traditional methods, such as [La] and gas exchange analysis, as well as a real noninvasive alternative to routine applications. The main methods for identifying the HRVTs are frequency domain $[10,12,13]$, time domain $[5,9,15]$, and nonlinear $[10,14]$ analysis. Success in identifying HRVTs and consequent estimation of AeT and AnT have been confirmed in different situations, such as in running [10-12], cycling $[5,9,13-15]$, and ski mountaineering $[16,17]$, and also in high-level swimmers [18], trained boys [10], obese adolescents [19], and individuals with type 2 diabetes [20].

The Poincaré plot is a nonlinear HRV analysis method that uses time domain markers [21] and is an important research area, since it allows its use in nonstationary data, a characteristic inherent to HRV, especially during the increase of effort intensity [22]. The Poincaréplot analysis provides the calculation of the standard deviation of instantaneous (SD1) and continuous long-term RR intervals (SD2) [1]. The SD1 marker has been shown to correlate strongly with vagal tone (PNS), and previous studies have pointed to an abrupt point of change in their behavior in intensities related to AeT $[2,5,9,11]$. SD2 marker has been shown to correlate with the PNS and SNS, and this variable shows a nonlinear pattern in intensities close to heavy and severe domains $[2,11]$. The applicability and efficacy of the Poincaré plot in the estimation of the AeT and AnT have been confirmed in previous studies in running [11] and cycling [9, 15, 23].

However, in addition to the heart rate (HR) which presents theoretical support for a nonlinear pattern, especially in intensity close to AnT [24], some aspects need to be better elucidated when using HRV markers for the estimation of AeT and AnT. Firstly, it would be the validity of the method since the majority of studies used visual analysis for HRVT identification [9-12, 16], which is influenced by the subjective aspect and experience of the evaluator. In order to remedy this limitation, Cheng et al. [25] proposed the Dmax method to identify the lactate and ventilatory thresholds. Previous studies demonstrated greater reliability of the Dmax method than visual analysis or the use of fixed [La] [26]. The Dmax method presents an important advantage which a breakpoint can always be detected [25], although a maximal test is needed. Only one study of our knowledge used the Dmax method to identify HRVTs [23]. Their results surprisingly on the contrary, as reported by the authors, pointed to the visual analysis as better indicators of reliability in the SD1 and RMSSD (square root of the mean squared differences of successive RR intervals) markers than the Dmax method. This way, it is doubted if the Dmax method is better or not than the visual analysis for the identification of AeT or AnT, when using HRV dataset. Nevertheless, the results of the aforementioned study [23] were not compared with traditional methods to estimate AeT and AnT, as [La] or gaseous exchanges; therefore, greater inferences are limited. Secondly, no study to date has analyzed the possibility to identify the HRVTs by the Dmax method in the different situations and conditions compared to lactate and ventilatory thresholds. Finally, the reproducibility of the method must be verified in relation to different situations and conditions, since it has only been tested on the cycle $[15,23]$.

The identification of HRVTs and consequently the estimation of MLSS can be a framework very important to control and monitor training workloads, as well as to assess the improvement in performance during an endurance training program [21]. The applicability of HRV thresholds in a single-day test perhaps can be very attractive for research studies of sports science, trainers, and practitioners users. Therefore, the aim of the study is to investigate the application of the Dmax method originally proposed by Cheng et al. [25], on HRV dataset to estimate the AeT and AnT, during a maximal incremental running test (MIRT) in male runners. Firstly, the hypothesis is that the HRVT identified by SD1 marker (HRVT DMAX1 $_{1}$ ) could be used to estimate the AeT, since this marker has been shown to correlate with the PNS $[2,9,11,15]$. Secondly, the hypothesis is that the HRVT identified by SD2 marker (HRVT DMAX2 $_{\text {) }}$ could be used to estimate AnT, since this marker has been shown to correlate with a significant PNS and mainly with the SNS [11]. The reproducibility of the HRVT DMAX1 $_{\text {and }}$ HRVT $_{\text {DMAX2 }}$, as well as AeT and AnT, will be verified.

\section{Materials and Methods}

2.1. Participants. Nineteen male recreational long-distance runners $(30.4 \pm 4.0$ years; body mass of $74.3 \pm 8.4 \mathrm{~kg}$; height of $176 \pm 6.3 \mathrm{~cm}$; body fat of $13.8 \pm 4.5 \%$ ) volunteered to participate in this study. All participants were healthy, without cardiovascular or orthopedic problems, nonsmokers, and not taking any medication. The study protocol complied with the Declaration of Helsinki for human experimentation [27] and was approved by the Institutional Ethics Committee of the University of São Paulo.

2.2. Study Design. All the participants performed two MIRTs interspersed by a washout period of 3-7 days. The tests were performed at the same time of the day and in standard laboratory conditions (humidity of $\approx 50 \%$ and temperature of $\approx 22^{\circ} \mathrm{C}$ ). Participants were instructed to avoid intense exercises, alcohol, and caffeine beverages 24 hours before each test and to consume a light meal 3 hours before the tests.

2.3. Maximal Incremental Running Test Protocol. Before MIRT, participants used a cardio belt for beat-to-beat heart rate (HR) measures (S810 Polar ${ }^{\circledR}$, Kempele, Finland), during rest for $20 \mathrm{~min}$ (10 min supine $+10 \mathrm{~min}$ sitting) for baseline measures of HR, HRV, and [La]. The [La] was obtained from a $25 \mu \mathrm{L}$ blood, drawn from the tip of the forefinger, and blood samples were then stored in Eppendorf tubes containing $50 \mu \mathrm{L}$ of $1 \% \mathrm{NaF}$ in a $-30^{\circ} \mathrm{C}$ environment, according to the recommendations of the manufacturer (YSI 1500 Sport, Yellow Springs, OH, USA). Later, the samples were analyzed using enzyme electrode technology (YSI 1500 Sport, Yellow Springs, OH, USA). Then, the participants were directed on the treadmill (CEFISE TK35, Nova Odessa, Brazil) and warmed up for $3 \mathrm{~min}$ at $5 \mathrm{~km} \cdot \mathrm{h}^{-1}$ and $1 \%$ gradient. The test started at $8 \mathrm{~km} \cdot \mathrm{h}^{-1}$, with $1 \mathrm{~km} \cdot \mathrm{h}^{-1}$ increases 
every $3 \mathrm{~min}$, until exhaustion, being a protocol adapted by Heck et al. [28]. The HR dataset was recorded continuously throughout the tests. Blood samples of $25 \mu \mathrm{L}$ were collected during the last $30 \mathrm{~s}$ of every stage, while the participant was running.

\subsection{Determination of Aerobic and Anaerobic Thresholds}

2.4.1. Heart Rate Variability Thresholds. The Dmax method was used to analyze the behavior of SD1 and SD2 markers, to identify the $\mathrm{HRVT}_{\mathrm{DMAX} 1}$ and $\mathrm{HRVT}_{\mathrm{DMAX} 2}$, respectively (Figure 1). The Dmax method was determined according to Cheng et al. [25], thereby providing individualized lactate threshold values according to the following equation:

$Y=a_{3} x^{3}+a_{2} x^{2}+a_{1} x+a_{0}, \quad$ where $x$ is the workload $\left(\mathrm{km} \cdot \mathrm{h}^{-1}\right)$,

where $Y$ represents the predicted values of SD1 or SD2 at a given workload $\left(\mathrm{km} \cdot \mathrm{h}^{-1}\right) ; a_{3}, a_{2}, a_{1}$, and $a_{0}$ are the intercepts; and $x$ is the speed. Briefly, the Dmax method reflects the longest perpendicular distance between SD1 and SD2 values predicted by a third-order polynomial function over actual (SD1 and SD2) values and values derived from a linear regression calculated with the first and last values of each curve, respectively. The SD1 marker was used because it has been shown to correlate strongly with vagal tone (PNS), and previous studies have pointed to an abrupt point of change in their behavior in intensities related to AeT $[2,5,9,11,15]$. SD2 marker has been shown to correlate with the PNS and SNS, and this variable shows a nonlinear pattern in intensities close to heavy and severe domains, being these related to AnT $[2,11]$. Thereafter, raw RR intervals were recorded during the last $60 \mathrm{~s}$ of each stage of the exercise, and then the Dmax method was applied on the measured values.

2.4.2. Lactate Thresholds. The first lactate threshold $\left(\mathrm{LT}_{1}\right)$ (i.e., AeT) was determined as the lowest value of the ratio [La]/speed [29]. After, the second lactate threshold $\left(\mathrm{LT}_{2}\right)$ (i.e. AnT) was determined as the running speed at $1.5 \mathrm{mmol} \cdot \mathrm{L}^{-1}$ above $\mathrm{LT}_{1}$ (Figure 1) [29]. The $\mathrm{LT}_{1}$ and $\mathrm{LT}_{2}$ derived from the Dickhuth et al.'s [29] methods were used as criterium measures, because $\mathrm{LT}_{1}$ has a high correlation with MLSS [7], and $\mathrm{LT}_{2}\left(+1.5 \mathrm{mmol} \cdot \mathrm{L}^{-1}\right)$ was used because it showed a high concordance with MLSS in runners during the MIRT with stages of $3 \mathrm{~min}$ [30].

All the thresholds, $\mathrm{HRVT}_{\mathrm{DMAX} 1}, \mathrm{HRVT}_{\mathrm{DMAX} 2}, \mathrm{LT}_{1}$, and $\mathrm{LT}_{2}$, were expressed as absolute and relative values for speed $\left(\mathrm{km} \cdot \mathrm{h}^{-1}\right)$, [La] $\left(\mathrm{mmol} \cdot \mathrm{L}^{-1}\right)$, milliseconds beat-to-beat $\mathrm{RR}$ intervals (ms), and HR (bpm).

2.5. Statistical Procedures. Values were expressed as mean and standard deviation $( \pm S D)$. After ensuring Gaussian data distribution (normality and homoscedasticity), a spreadsheet was used for the analysis of concurrent validity [31] and statistical standards were followed [32]. Cohen's [33] (d) effect sizes and ANOVA with Scheffe's post hoc test were used to compare the magnitude of the differences between the thresholds $\mathrm{LT}_{1}$ and $\mathrm{LT}_{2}$ with $\mathrm{HRVT}_{\mathrm{DMAX}}$ and $\mathrm{HRVT}_{\mathrm{DMAX} 2}$, respectively. Additionally, the standard error of the estimate (SEE), the bias $\pm 95 \%$ of limits of agreement [LoA] of the Bland and Altman analysis [34], and the Pearson product-moment correlation were used to evaluate the association between the different methods for identifying thresholds. For measures, reliability determination, the intraclass correlation coefficient (ICC), and the typical error of measurement (TEM) were performed using a Hopkins spreadsheet [31]. The $d$ values were interpreted using the following scale: $<0.20$ (trivial), $0.2-0.6$ (small), $0.6-1.2$ (moderate), 1.2-2.0 (large), 2.0-4.0 (very large), and $>4.0$ (extremely large) [33]. Additionally, the ICC and the Pearson correlation coefficients $(r)$ were interpreted as follows: $<0.10$ (trivial), 0.30 (small), 0.50 (moderate), 0.70 (large), 0.90 (very large), 0.99 (nearly perfect), and 1 (perfect) [31]. The data analysis was performed using the SPSS (19.0). The significance adopted was set at $p<0.05$.

\section{Results}

3.1. Identification of $H R V T_{D M A X 1}, H R V T_{D M A X 2}, L T_{1}$, and $L T_{2}$. No significant differences were observed, neither for absolute nor for relative values, between $\mathrm{HRVT}_{\mathrm{DMAX} 1}$ and $\mathrm{LT}_{1}$ when expressed for speed $(p=0.55 ; d=0.45$ and $p=0.10 ; d=0.76)$, lactate $(p=0.24 ; d=0.79$ and $p=0.13$; $d=0.06)$, RR $(p=0.54 ; d=0.48$ and $p=0.50 ; d=0.49)$, and HR ( $p=0.45 ; d=0.44$ and $p=0.30 ; d=0.51)$, respectively. In the same way, significant differences were not observed between $\mathrm{HRVT}_{\mathrm{DMAX} 2}$ and $\mathrm{LT}_{2}$ when expressed for lactate $(p=0.13 ; d=0.14$ and $p=0.99 ; d=0.00)$ and $\mathrm{RR}(p=0.22$; $d=0.68$ and $p=0.23 ; d=0.68)$, but on the other hand, significant differences were observed when expressed for speed $(p=0.00 ; d=1.21$ and $p=0.00 ; d=1.9)$ and HR $(p=0.02 ; d=1.15$ and $p=0.00 ; d=1.24)$, respectively. Further, no significant differences were observed, neither for absolute nor for relative values, between $\mathrm{HRVT}_{\mathrm{DMAX} 2}$ and $\mathrm{LT}_{1}$ when expressed for speed $(p=0.66 ; d=0.41$ and $p=0.16 ; d=0.75), \operatorname{RR}(p=0.73 ; d=0.35$ and $p=0.66$; $d=0.70)$, and $\operatorname{HR}(p=0.61 ; d=0.33$ and $p=0.61 ; d=0.39)$. Table 1 shows the results of all the methods.

Table 2 shows in detail the results of the Pearson correlation coefficient between the methods expressed as absolute and relative values for speed, lactate, RR, and HR.

Figure 2 shows the magnitude of differences between HRVT $_{\text {DMAX1 }}$ and $\mathrm{LT}_{1}$, and HRVT $\mathrm{DMAX}_{2}$ and $\mathrm{LT}_{2}$. The Bland-Altman and regression analysis showed between $\mathrm{HRVT}_{\text {DMAX1 }}$ and $\mathrm{LT}_{1}$ the bias $\pm \mathrm{LoA}=0.84 \pm 3.7 \mathrm{~km} \cdot \mathrm{h}^{-1}$ and $\mathrm{SEE}=1.2 \mathrm{~km} \cdot \mathrm{h}^{-1} \quad(95 \%$ CI, 0.9-1.9), and between $\mathrm{HRVT}_{\text {DMAX2 }}$ and $\mathrm{LT}_{2}$ the bias $\pm \mathrm{LoA}=-1.07 \pm 1.8 \mathrm{~km} \cdot \mathrm{h}^{-1}$ and SEE $=1.1 \mathrm{~km} \cdot \mathrm{h}^{-1}(95 \% \mathrm{CI}, 0.8-1.6)$.

3.2. Reliability of the HRV and [La] Thresholds. In relation to baseline HRV values $(423.0 \pm 28 \mathrm{~ms}$ vs. $425.7 \pm 25 \mathrm{~ms}$; $p=0.95 ; d=0.09)$ and baseline lactate values $(1.34 \pm 0.4$ $\mathrm{mmol} \cdot \mathrm{L}^{-1}$ vs. $\left.1.25 \pm 0.3 \mathrm{mmol} \cdot \mathrm{L}^{-1} \cdot p=0.77 ; d=0.41\right)$, no significant differences were observed between test and retest. With regard to values recorded at the exhaustion in MIRT, 


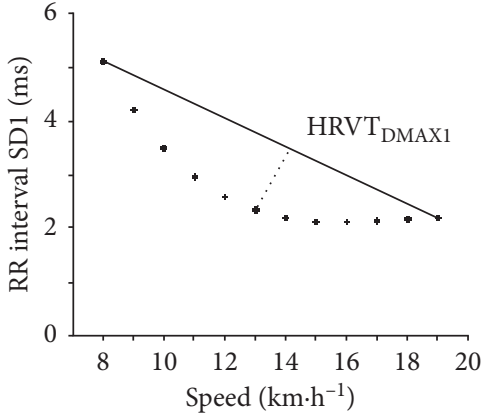

(a)

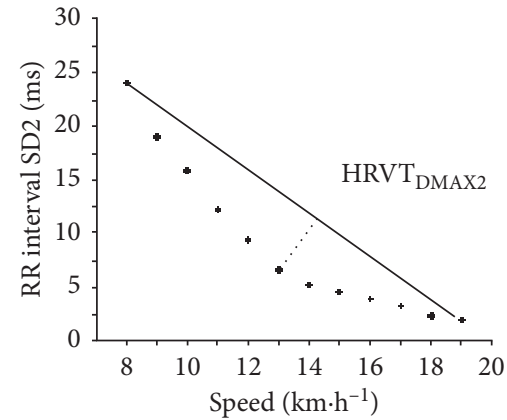

(b)

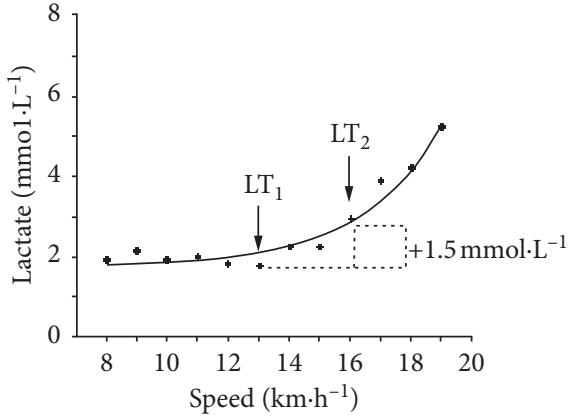

(c)

FIgURE 1: The HRVT $\operatorname{DMAX}_{1}$ (a) and $\operatorname{HRVT}_{\mathrm{DMAX} 2}$ (b) thresholds determined by the Dmax method and $\mathrm{LT}_{1}$ and $\mathrm{LT}_{2}$ (c) determined by lactate concentrations.

TABLE 1: Aerobic and anaerobic thresholds expressed as absolute and relative (mean \pm SD) values for speed, blood lactate concentrations, milliseconds beat-to-beat RR intervals, and heart rate during maximal running test among trained male runners.

\begin{tabular}{|c|c|c|c|c|c|c|c|c|}
\hline & \multirow{2}{*}{$\begin{array}{c}\text { Speed } \\
\left(\mathrm{km} \cdot \mathrm{h}^{-1}\right)\end{array}$} & \multicolumn{4}{|c|}{ Lactate } & \multirow{2}{*}{$\begin{array}{l}\mathrm{RR} \\
\%^{\mathrm{b}}\end{array}$} & \multicolumn{2}{|c|}{ HR } \\
\hline & & $\%^{\mathrm{a}}$ & $\left(\mathrm{mmol} \cdot \mathrm{L}^{-1}\right)$ & $\%^{\mathrm{a}}$ & $(\mathrm{ms})$ & & (bpm) & $\%^{\mathrm{a}}$ \\
\hline $\begin{array}{l}\text { HRVT }_{\text {DMAX1 }} \\
(95 \% \text { CI })\end{array}$ & $\begin{array}{l}12.1 \pm 1.4^{* *} \\
(11.4-12.7)\end{array}$ & $\begin{array}{l}73.4 \pm 7.3^{* *} \\
(69.9-77.0)\end{array}$ & $\begin{array}{l}2.2 \pm 0.8 \\
(1.8-2.6)\end{array}$ & $\begin{array}{c}32.7 \pm 9.4 \\
(28.2-37.2)\end{array}$ & $\begin{array}{c}379.4 \pm 38 \\
(360.9-398.0)\end{array}$ & $\begin{array}{c}54.1 \pm 5.2 \\
(51.6-56.6)\end{array}$ & $\begin{array}{l}159 \pm 15^{*} \\
(151-167)\end{array}$ & $\begin{array}{l}83.0 \pm 7.7^{*} \\
(79.3-86.7)\end{array}$ \\
\hline HRVT $_{\text {DMAX2 }}$ & $12.0 \pm 1.2^{* *}$ & $72.9 \pm 7.4^{* *}$ & $2.3 \pm 1.2$ & $33.4 \pm 15.0$ & $383.6 \pm 41$ & $54.6 \pm 4.5$ & $158 \pm 17^{*}$ & $82.2 \pm 7.9^{* * *}$ \\
\hline$(95 \% \mathrm{CI})$ & $(11.3-12.6)$ & $(69.3-76.5)$ & $(1.7-2.9)$ & $(26.1-40.7)$ & $(363.8-40$ & $(52.4$ & $(149$ & $(78.4-86.1)$ \\
\hline $\begin{array}{l}\mathrm{LT}_{1} \\
(95 \% \mathrm{CI})\end{array}$ & $\begin{array}{l}11.2 \pm 2.1^{* *} \\
(10.2-12.3)\end{array}$ & $\begin{array}{l}67.7 \pm 7.6^{* *} \\
(64.1-71.4)\end{array}$ & $\begin{array}{c}1.7 \pm 0.5^{* *} \\
(1.4-2.0)\end{array}$ & $\begin{array}{c}24.7 \pm 8.5 \\
(20.6-28.8)\end{array}$ & $\begin{array}{c}397.9 \pm 38^{* *} \\
(379.3-416.5)\end{array}$ & $\begin{array}{l}56.8 \pm 5.6^{* *} \\
(54.0-59.5)\end{array}$ & $\begin{array}{l}151 \pm 14^{* *} \\
(144-158)\end{array}$ & $\begin{array}{l}78.8 \pm 6.3^{* *} \\
(75.8-81.9)\end{array}$ \\
\hline $\mathrm{LT}_{2}$ & $14.1 \pm 2.1$ & $85.2 \pm 4.9$ & $2.9 \pm 0.5$ & $33.5 \pm 6.2$ & $356.7 \pm 37$ & $51.0 \pm 5.9$ & $173 \pm 9$ & $90.0 \pm 3.8$ \\
\hline$(95 \% \mathrm{CI})$ & $(13.0-15.1)$ & $(82.8-87.5)$ & $(2.7-3.2)$ & $(30.5-36.5)$ & $(338.4-375.0)$ & $(48.1-53.8)$ & $(168-177)$ & (88.2-91.9) \\
\hline
\end{tabular}

a Percentage values are relative to maximal values during the MIRT. ${ }^{b}$ Percentage values are relative to $5 \mathrm{~km} \cdot \mathrm{h}^{-1}$ values $(\mathrm{RR}) ; \mathrm{CI}=$ confidence interval. ${ }^{* *} p<0.01$ and ${ }^{*} p<0.05$ in relation to $\mathrm{LT}_{2}$ method.

TABle 2: Pearson correlation expressed as absolute and relative values for speed, blood lactate, RR interval, and heart rate during maximal running test among well-trained runners.

\begin{tabular}{|c|c|c|c|c|c|c|c|c|c|c|c|c|c|c|c|c|}
\hline & \multicolumn{4}{|c|}{ Speed } & \multicolumn{4}{|c|}{ Lactate } & \multicolumn{4}{|c|}{ RR } & \multicolumn{4}{|c|}{ HR } \\
\hline & I & II & III & IV & $\mathrm{I}$ & II & III & IV & $\mathrm{I}$ & II & III & IV & $\mathrm{I}$ & II & III & IV \\
\hline $\begin{array}{l}\mathrm{HRVT}_{\mathrm{DMAX} 1} \\
\text { (I) }\end{array}$ & 1 & 0.44 & $0.46^{*}$ & $0.58^{* *}$ & 1 & 0.42 & $0.55^{*}$ & $0.66^{* *}$ & 1 & $0.83^{* *}$ & 0.31 & 0.19 & 1 & $0.85^{* *}$ & 0.45 & $062 *$ \\
\hline $\begin{array}{l}\mathrm{HRVT}_{\mathrm{DMAX} 2} \\
\text { (II) }\end{array}$ & 0.29 & 1 & 0.26 & $0.48^{*}$ & 0.42 & 1 & $0.67^{* *}$ & 0.35 & $0.78^{* *}$ & 1 & 0.44 & 0.24 & $0.81^{* *}$ & 1 & $0.52^{*}$ & $0.71^{* *}$ \\
\hline $\mathrm{LT}_{1}(\mathrm{III})$ & -0.18 & $-0.46^{*}$ & 1 & $0.85^{* *}$ & $0.45^{*}$ & $0.61^{* *}$ & 1 & $0.59^{* *}$ & 0.38 & 0.38 & 1 & $0.54^{*}$ & 0.29 & 0.33 & 1 & $0.81^{* *}$ \\
\hline $\mathrm{LT}_{2}(\mathrm{IV})$ & -0.06 & -0.27 & 0.41 & 1 & 0.29 & -0.00 & -0.08 & 1 & 0.36 & 0.28 & $0.66^{* *}$ & 1 & $0.57^{* *}$ & $0.55^{*}$ & $0.54^{*}$ & 1 \\
\hline
\end{tabular}

$\mathrm{HRVT}_{\mathrm{DMAX} 1}=\mathrm{I} ; \mathrm{HRVT}_{\mathrm{DMAX} 2}=\mathrm{II} ; \mathrm{LT}_{1}=\mathrm{III}$, and $\mathrm{LT}_{2}=\mathrm{IV}$. Upper and lower triangles of each variable (speed, lactate, RR, and $\mathrm{HR}$ ) refer to absolute and relative values, respectively. ${ }^{*} p<0.05 .{ }^{* *} p<0.01$

no significant differences were observed between test and retest for speed $\left(16.4 \pm 1.7 \mathrm{~km} \cdot \mathrm{h}^{-1}\right.$ vs. $16.6 \pm 1.7 \mathrm{~km} \cdot \mathrm{h}^{-1}$; $p=0.96 ; d=0.09), \operatorname{HR}(192 \pm 5$ bpm vs. $191 \pm 5$ bpm; $p=0.98 ; \quad d=0.12)$, and $[\mathrm{La}] \quad\left(9.2 \pm 1.8 \mathrm{mmol} \cdot \mathrm{L}^{-1}\right.$ vs. $\left.8.2 \pm 1.9 \mathrm{mmol} \cdot \mathrm{L}^{-1} ; p=0.88 ; d=0.52\right)$, respectively.

Significant differences were not observed between test and retest for all thresholds $\left(\mathrm{HRVT}_{\mathrm{DMAX} 1}=12.1 \pm 1.4 \mathrm{~km} \cdot \mathrm{h}^{-1}\right.$ vs. $12.3 \pm 1.5 \mathrm{~km} \cdot \mathrm{h}^{-1}, \quad \operatorname{HRVT}_{\mathrm{DMAX} 2}=12.0 \pm 1.2 \mathrm{~km} \cdot \mathrm{h}^{-1}$ vs. $11.7 \pm 1.4 \mathrm{~km} \cdot \mathrm{h}^{-1}, \mathrm{LT}_{1}=11.2 \pm 2.1 \mathrm{~km} \cdot \mathrm{h}^{-1} \mathrm{vs.} 12.0 \pm 1.5 \mathrm{~km} \cdot \mathrm{h}^{-1}$, and $\mathrm{LT}_{2}=14.1 \pm 2.1 \mathrm{~km} \cdot \mathrm{h}^{-1}$ vs. $14.2 \pm 1.7 \mathrm{~km} \cdot \mathrm{h}^{-1}$ ). Large ICCs were found to $\mathrm{HRVT}_{\text {DMAX1 }}$ when expressed in relation to RR and HR (ICC $=0.80-0.81 ;$ TEM $=5.1-4.9 \%$, respectively). The Bland-Altman and regression analysis showed the bias $\pm \mathrm{LoA}=0.84 \pm 3.7 \mathrm{~km} \cdot \mathrm{h}^{-1}$. In the same way, large ICCs were found to $\mathrm{HRVT}_{\mathrm{DMAX} 2}$ when expressed in relation to $\mathrm{RR}$ and HR ( $\mathrm{ICC}=0.80-0.82 ; \mathrm{TEM}=5.5-5.6 \%$, respectively). Results further suggested a consistent reproducibility for $\mathrm{LT}_{1}$ and $\mathrm{LT}_{2}$, since the large to nearly perfect ICCs were showed for speed and HR measures (ICC $=0.90-0.97$ and $0.80-0.94$; $\mathrm{TEM}=6.4-4.9 \%$ and $2.8-1.7 \%$, respectively). Table 3 shows all results of ICC and TEM for all the methods (Figure 3).

\section{Discussion}

The main findings of the present study were that the application of the Dmax method on HRV dataset (SD1 and 


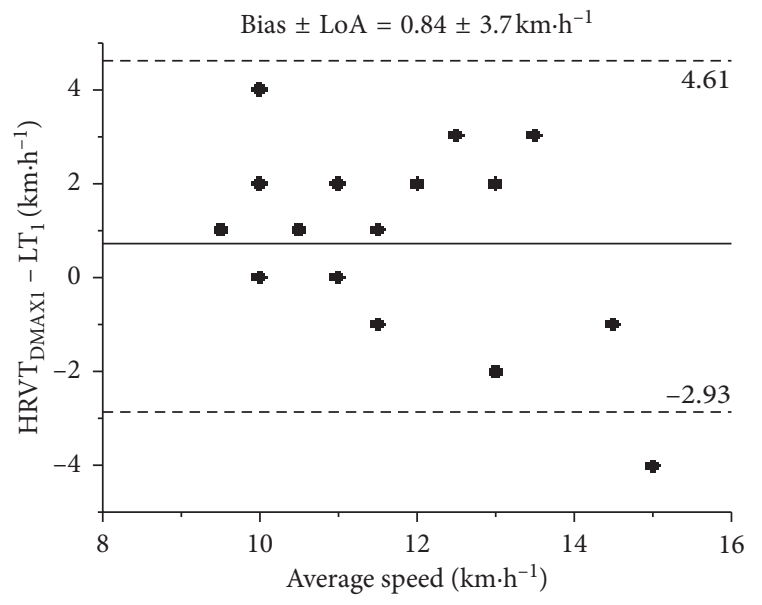

(a)

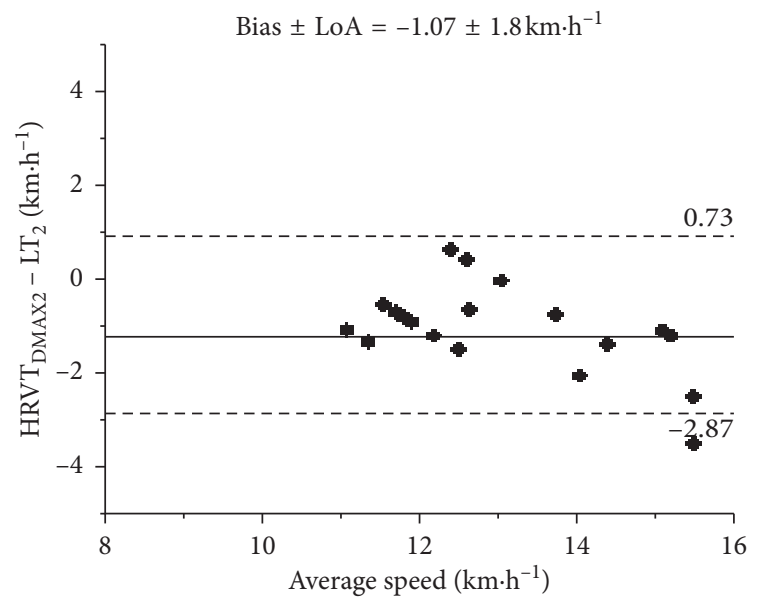

(c)

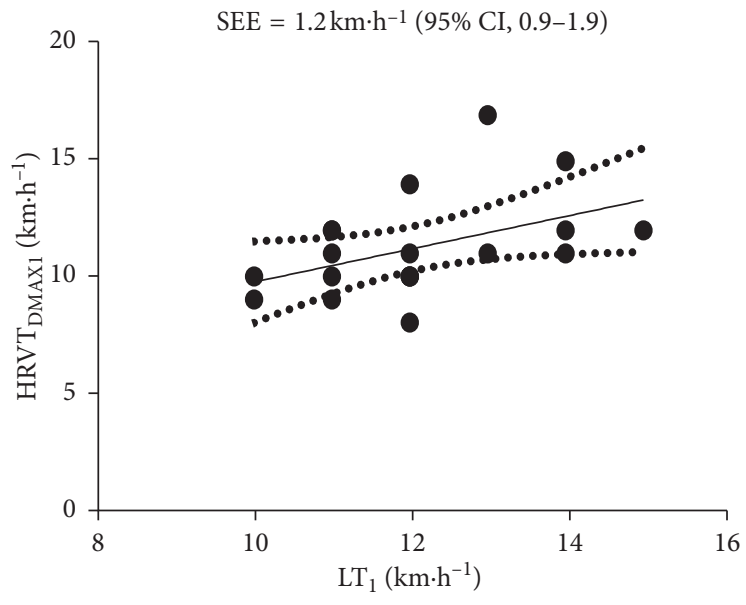

(b)

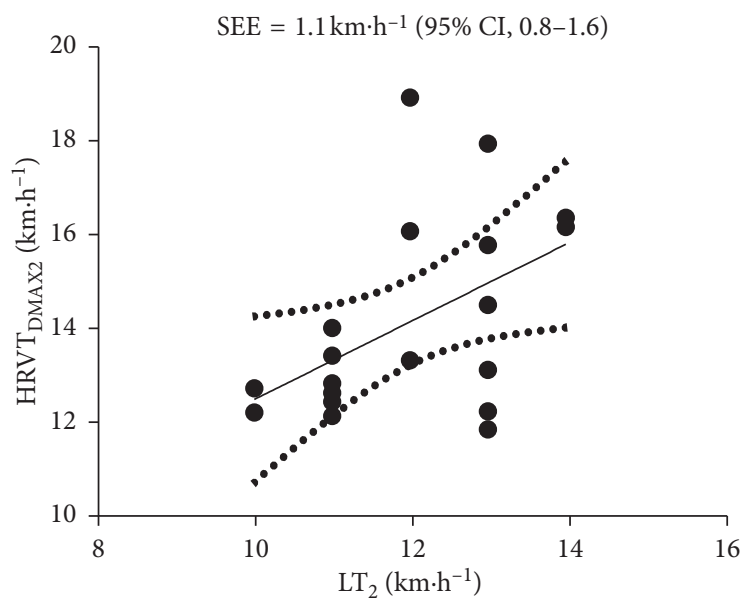

(d)

FIGURE 2: Bland and Altman plot for the agreement between $\mathrm{HRVT}_{\mathrm{DMAX} 1}$ and $\mathrm{LT}_{1}\left(\mathrm{a}\right.$ ), and $\mathrm{HRVT}_{\mathrm{DMAX} 2}$ and $\mathrm{LT}_{2}$ (c), with bias (continuous line) and the $95 \%$ limits of agreement (discontinuous lines) for speed measures. The relationship between $\mathrm{HRVT}_{\mathrm{DMAX}}$ for speed and $\mathrm{LT}_{1}(\mathrm{~b})$ and $\mathrm{HRVT}_{\mathrm{DMAX} 2}$ and $\mathrm{LT}_{2}(\mathrm{~d})$ measures; solid and dashed lines represent the regression line and the $95 \%$ confidence intervals, respectively.

TABLE 3: Reliability analysis for HRVT $\mathrm{DMAX}_{1}, \mathrm{HRVT}_{\mathrm{DMAX} 2}, \mathrm{LT}_{1}$, and $\mathrm{LT}_{2}$.

\begin{tabular}{|c|c|c|c|c|c|c|c|c|c|c|c|c|c|c|c|c|}
\hline & \multicolumn{4}{|c|}{ Speed } & \multicolumn{4}{|c|}{ Lactate } & \multicolumn{4}{|c|}{$\mathrm{RR}$} & \multicolumn{4}{|c|}{ HR } \\
\hline & \multicolumn{2}{|c|}{ ICC } & \multicolumn{2}{|c|}{ TEM $^{\mathrm{a}}$} & \multicolumn{2}{|c|}{ ICC } & \multicolumn{2}{|c|}{ TEM $^{\mathrm{a}}$} & \multicolumn{2}{|c|}{ ICC } & \multicolumn{2}{|c|}{ TEM $^{\mathrm{a}}$} & \multicolumn{2}{|c|}{ ICC } & \multicolumn{2}{|c|}{ TEM $^{\mathrm{a}}$} \\
\hline & $M$ & $p$ & $\mathrm{~km} \cdot \mathrm{h}^{-1}$ & $\%$ & $M$ & $p$ & $\mathrm{mmol} \cdot \mathrm{l}^{-1}$ & $\%$ & $M$ & $p$ & $\mathrm{~ms}$ & $\%$ & $M$ & $p$ & bpm & $\%$ \\
\hline HRVT & 0.48 & 0.08 & 1.18 & 9.6 & 0.42 & 0.12 & 0.67 & 29.6 & 0.80 & 0.00 & 19.4 & 5.1 & 0.81 & 0.00 & 8 & 4.9 \\
\hline HRVT $_{\text {DMAX2 }}$ & 0.30 & 0.22 & 1.21 & 10.1 & 0.60 & 0.29 & 0.80 & 36.4 & 0.80 & 0.00 & 21.8 & 5.6 & 0.82 & 0.00 & 8 & 5.5 \\
\hline $\mathrm{LT}_{1}$ & 0.90 & 0.00 & 0.75 & 6.4 & 0.73 & 0.00 & 0.33 & 19.2 & 0.75 & 0.00 & 21.6 & 5.5 & 0.80 & 0.00 & 7 & 4.9 \\
\hline $\mathrm{LT}_{2}$ & 0.97 & 0.00 & 0.40 & 2.8 & 0.71 & 0.00 & 0.32 & 10.6 & 0.49 & 0.08 & 24.2 & 6.9 & 0.94 & 0.00 & 2 & 1.7 \\
\hline
\end{tabular}

${ }^{a}$ Multiply and divide these values by 1.4 to obtain the $95 \%$ confidence intervals $(x / \div 1.4)$. ICC, intraclass coefficient correlation; TEM, typical error of measurement.

SD2 markers by the Poincare plot, being $\mathrm{HRVT}_{\mathrm{DMAX} 1}$ and $\mathrm{HRVT}_{\text {DMAX2}}$, respectively) enabled the estimation of the $\mathrm{LT}_{1}$ during a MIRT in male runners. However, the results refute one of the hypotheses of the study, which was that the HRVT $_{\text {DMAX2 } 2}$ method would estimate the AnT. The relative values found in $\mathrm{HRVT}_{\mathrm{DMAX} 2}$ and $\mathrm{HRVT}_{\mathrm{DMAX} 1}$ showed values with a greater approximation to the AeT. Consequently, it seems to suggest that when the Dmax method is applied to a HRV dataset extracted by the Poincare plot, it is possible to identify a transition zone, with an approximation to the AeT, being an important intensity to improve cardiorespiratory and neuromuscular responses of runners. Furthermore, the results also suggested a consistent reproducibility for $\mathrm{LT}_{1}$ and $\mathrm{LT}_{2}$ (ICC $=0.90$ and 0.97 , respectively), as well as, moderate to $\mathrm{HRVT}_{\text {DMAX1 }}$ $($ ICC $=0.48)$ with low bias $\left(-0.18 \pm 3.4 \mathrm{~km} \cdot \mathrm{h}^{-1}\right)$. 


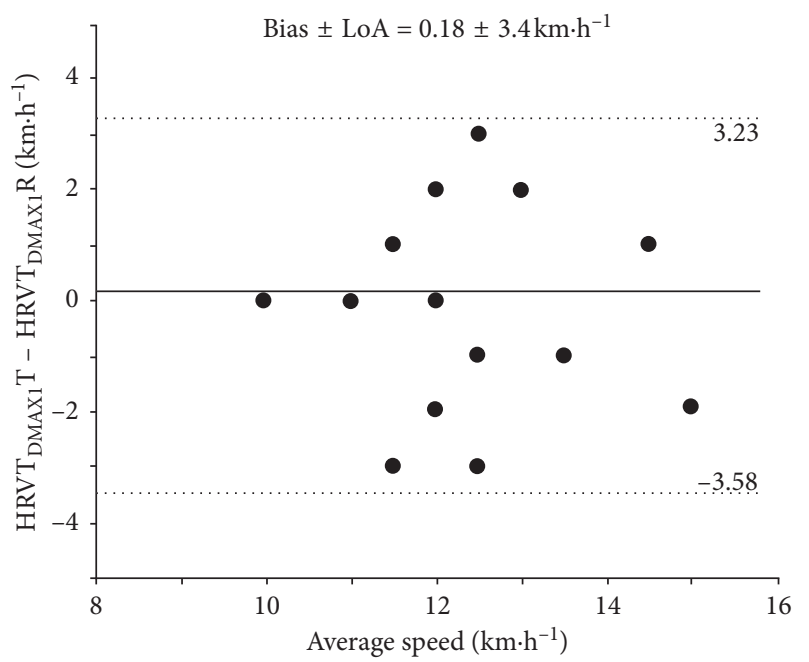

(a)

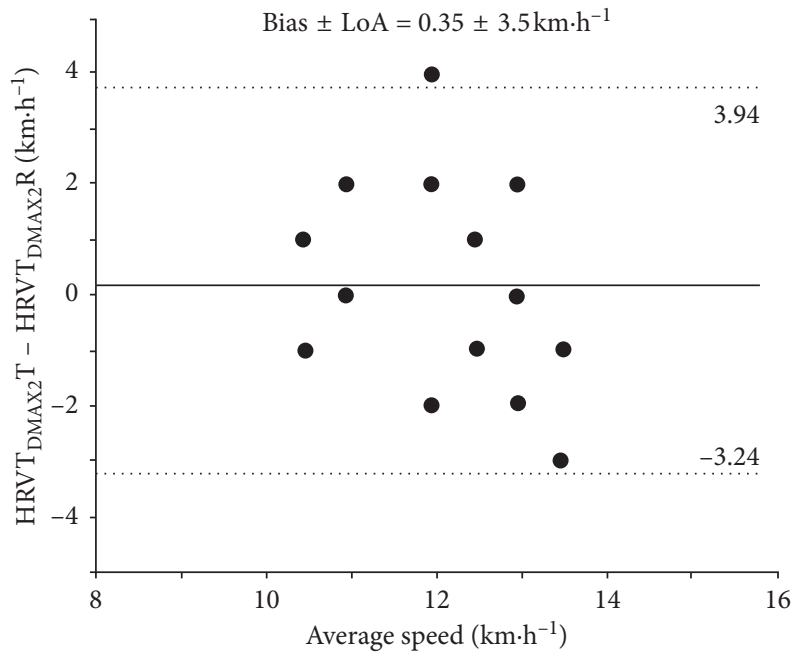

(b)

FIgURE 3: Bland and Altman plot for the reproducibility of $\operatorname{HRVT}_{\mathrm{DMAX} 1}$ (a) and $\operatorname{HRVT}_{\mathrm{DMAX} 2}$ (b) methods.

The results of the SD1 marker, which was used to identify the $\mathrm{HRVT}_{\text {DMAX1}}$, showed values of approximately $\approx 73.4 \%$ of the peak speed value, being these intensities related to the AeT $[3,4]$. However, the values were slightly above the values reported in previous studies such as Garcia-Tabar et al. [9] analyzing a homogeneous group of professional male worldclass road cyclists $\left(\approx 36-52 \% \mathrm{~W}_{\mathrm{PEAK}}\right)$, Candido et al. [23] analyzing healthy individuals $\left(\approx 50-60 \% \mathrm{~W}_{\mathrm{PEAK}}\right)$, Sales et al. [20] analyzing individuals with type 2 diabetes $(\approx 64-66 \%$ $\mathrm{VO}_{2 \mathrm{PEAK}}$ ), and Tulppo et al. [2] investigating complete or not parasympathetic blockade. The HRVT DMAX1 $_{1}$ has elicited significant correlation when compared to $\mathrm{LT}_{1}(r=0.46)$. The results of the present study in relation to $\mathrm{HRVT}_{\mathrm{DMAX} 1}$ are slightly below those found by Garcia-Tabar et al. [9], which used the same marker of PNS (SD1) to estimate the LT $(r=0.66-0.88)$, although different protocols and methodologies were applied. However, it is important to note that only a study of our knowledge by Nascimento et al. [11] used the same ergometer when analyzing HRV indices by the Poincaré plot and [La], in case the treadmill, and all other studies used a cycle ergometer. Consequently, greater comparisons are limits due mainly to the specificity and differences in the movement gesture as well as in the recruitment of motor units $[35,36]$.

It is important to note that the SD1 marker, which presents the prevalence of PNS activity, correlates with indices representing high-frequency bands (HF), such as in the Fourier Transform, when analyzing the behavior of HRV by frequency domain [1]. In addition, studies suggest that the respiratory pattern has a strong effect on the HF-HRV bands, both at rest and at exercise [37-39]. During exercise in heavy domain occurs an increase in respiratory frequency with a constant final volume, triggering a mechanical effect on the sinus node, concomitant with an increase in HF. This can be demonstrated in previous studies which identified changes in HF behavior below and above of ventilatory threshold [37].
The SD2 marker used in the present study to identify the $\mathrm{HRVT}_{\text {DMAX2 }}$ showed significant differences in relation to $\mathrm{LT}_{2}$ when expressed for absolute and relative values of speed and $\mathrm{HR}$, but not when expressed to lactate and RR, respectively. On the other hand, it is important to note that HRVT $\mathrm{DMAX}_{2}$ has elicited moderate coefficient values $(r=0.48)$ and significant coefficient values $(r=0.71)$ when compared to $\mathrm{LT}_{2}$, with the values expressed for speed and HR, respectively. This variation in the correlations may be explained in part by the size and heterogeneous characteristic of the sample (body fat coefficient of variation (CV) is 33\%). The values found in relation to the $\mathrm{HRVT}_{\mathrm{DMAX} 2}$ were approximately $\approx 72.9 \%$ of the peak speed value, being these intensities related to the AeT $[3,4]$. Previous studies showed a breakpoint in SD2 to intensities of approximately $\approx 80 \%-90 \% \quad \mathrm{VO}_{2 \mathrm{MAX}}$ during maximal progressive cycling test [2] and intensities of approximately $\approx 86.1 \%$ of the peak speed value during MIRT [11]. However, as previously mentioned in relation to the possibility of identifying a single breakpoint by the Dmax method, these results suggest that the SD2 marker shows a nonlinearity behavior during a MIRT. Therefore, in addition to a breakpoint in intensity close to the AnT, as already demonstrated in previous studies $[2,11]$, a significant change point in SD2 also occurs at near intensities related to the AeT.

In the present study, the approximation of $\mathrm{HRVT}_{\mathrm{DMAX} 2}$ with the AeT, possibly, is due to the use of the Dmax method. This method allows the identification of only one breakpoint, although it is a nonsubjective method when compared to the visual method, for example. However, there are questions concerning the determination of the breakpoint by the Dmax method, in relation to the amount of values used in the model construction, being that the initial and final values can influence and compromise greater inferences when comparing the identification of the AeT or AnT [40].

Perhaps the use of different mathematical models [40] on HRV dataset, even with the possibility of submaximal tests 
[9], could allow greater accuracy in the estimation of AeT and $\mathrm{AnT}$ in different situations and populations involved. Moreover, both $\mathrm{HRVT}_{\mathrm{DMAX} 1}$ and $\mathrm{HRVT}_{\mathrm{DMAX} 2}$ are methods relatively simple to analyze and do not require a fixed number of RR intervals nor long recording periods [41], which facilitates the evaluation during incremental exercise. The usefulness of the HRVT $\mathrm{DMAX}_{\mathrm{DM}}$ and HRVT $_{\text {DMAX2 }}$ to estimate the AeT and determine aerobic capacity to prescribe exercise training intensities in sports performance and rehabilitation programs, from SD1 and SD2 values, is of great interest. The HRVTs may be objectively and noninvasively determined and are of lower cost than lactate or ventilatory threshold assessments, where blood lactate or gas analysis equipment is required as well as specialized professionals.

The $\mathrm{LT}_{1}$ and $\mathrm{LT}_{2}$ methods demonstrated a high level of reliability $(\mathrm{ICC}=0.90$ and $0.97 ; p<0.001$; TEM $=0.75$ and $0.40 \mathrm{~km} \cdot \mathrm{h}^{-1} ; \mathrm{TEM}=6.4$ and $2.8 \%$, respectively). These results presented lower values than previous studies using similar markers for determination of $\mathrm{LT}_{1}$ and $\mathrm{LT}_{2}(\mathrm{TEM}=2.8$ and $3.6 \%$, respectively) [42]. Bland and Altman presented low values for difference between the test and retest as a function of their mean (bias $\pm \mathrm{LoA}=-0.73 \pm 2.0 \mathrm{~km} \cdot \mathrm{h}^{-1}$; bias \pm LoA $=-0.08 \pm 1.1 \mathrm{~km} \cdot \mathrm{h}^{-1}$, respectively). Regarding the $\mathrm{HRVT}_{\mathrm{DMAX} 1}$, significant values were found in relation to RR and HR (ICC $=0.80$ and $0.81 ; p<0.001$, respectively), but on the other hand, moderate values were found when expressed in relation to speed $(\mathrm{ICC}=0.48)$, being below those found in a previous study $(\mathrm{ICC}=0.73)$ using the same marker for identification of AeT [15], but through visual identification instead of the Dmax method. Regarding the TEM, results were slightly above the values found in the aforementioned study $($ TEM $=8.5 \%)$. Bland and Altman presented low values for difference between the test and retest as a function of their mean (bias \pm LoA $=-0.18 \pm 3.4 \mathrm{~km} \cdot \mathrm{h}^{-1}$ ). Already to the $\mathrm{HRVT}_{\text {DMAX2}}$, significant values were found when expressed in relation to lactate (ICC $=0.60 ; p<0.05)$ and RR and HR (ICC $=0.80$ and $0.82 ; p<0.001$, respectively), with low values only when expressed in relation to speed $(\mathrm{ICC}=0.30$; $p<0.22$ ), corroborating previous studies that used HRV markers by the Dmax method [23].

Due to its low cost, noninvasive nature, and high applicability, HRVTs is an important framework for researchers, trainers, and race practitioners. In the present study, its simplified and nonsubjective identification by the Dmax method suggests the possibility of planning a training program with a safe intensity in a metabolic transition zone, being slightly above the values found for AeT.

The present study certainly has some limitations that must be considered in the analysis of the results and their applicability. Aspects such as heterogeneous characteristic of the sample may try to explain in part the variation of correlation values between the different methods, since previous studies report that the greater the heterogeneity of a group, the greater the magnitudes of correlation [32]. Another fact may be just the recruitment of male runners and the need to perform a MIRT. In addition, MLSS was not used as a gold standard, but $\mathrm{LT}_{2}$ had a good approximation of MLSS in runners [30]. Therefore, it is suggested to carry out studies with different characteristics of samples, such as gender, age, and levels of training.

\section{Conclusion}

To the best of our knowledge, this is the first report on the application of the Dmax method on a HRV dataset to identify the lactate thresholds, during a MIRT. The results of this study showed that the Dmax method applied over a set of HRV during a MIRT in male recreational long-distance runners allowed the identification of HRVTs approaching the AeT. The HRVTs are of low cost, noninvasive nature, and high applicability. A limiting factor for the interpretation of the data is the recruitment of only males and trained, which do not allow the generalization of results to different populations. Thus, further studies are needed to confirm the reproducibility of HRVT as well as its use in different protocols, genders, age groups, and levels of training.

\section{Disclosure}

The level of evidence is Level II Study of Diagnostic Test.

$\begin{array}{ll}\text { Abbreviations } \\ \text { HRV: } & \text { Heart rate variability } \\ \text { VO }_{2 M A X}: & \text { Maximum oxygen uptake } \\ \text { AeT: } & \text { Aerobic threshold } \\ \text { PNS: } & \text { Parasympathetic nervous system } \\ \text { SNS: } & \text { Sympathetic nervous system } \\ \text { AnT: } & \text { Anaerobic threshold } \\ \text { HRVT: } & \text { Heart rate variability threshold } \\ \text { SD1: } & \text { The standard deviation of instantaneous RR } \\ & \text { intervals } \\ \text { SD2: } & \text { The continuous long-term RR intervals } \\ \text { HR: } & \text { Heart rate } \\ \text { MIRT: } & \text { Maximal incremental running test } \\ \text { LA: } & \text { Lactate concentrations. }\end{array}$

\section{Data Availability}

The data used to support the findings of this study are available from the corresponding author upon request.

\section{Conflicts of Interest}

The authors declare no conflicts of interest regarding the publication of this manuscript.

\section{Acknowledgments}

This study was financed in part by the Coordenacão de Aperfeiçoamento de Pessoal de Nível Superior-Brasil (CAPES)-Finance Code 001. Dr. Paulo Cesar do Nascimento Salvador also acknowledges the support by grants from $\mathrm{CNPq}$, Conselho Nacional de Desenvolvimento Científico e Tecnológico-Brasil (154191/2018-3). 


\section{References}

[1] M. Malik, "Task force of the European society of cardiology and the north American society of pacing and electrophysiology. Heart rate variability: standards of measurement, physiological interpretation and clinical use," Circulation, vol. 93, no. 5, pp. 1043-1065, 1996.

[2] M. P. Tulppo, T. H. Makikallio, T. E. Takala, T. Seppanen, and H. V. Huikuri, "Quantitative beat-to-beat analysis of heart rate dynamics during exercise," American Journal of Physiology-Heart and Circulatory Physiology, vol. 271, no. 1, pp. H244-H252, 1996.

[3] O. Faude, W. Kindermann, and T. Meyer, "Lactate threshold concepts: how valid are they?," Sports Medicine, vol. 39, no. 6, pp. 469-490, 2009.

[4] J. S. Skinner and T. M. McLellan, "The transition from aerobic to anaerobic metabolism," Research Quarterly for Exercise and Sport, vol. 51, no. 1, pp. 234-248, 1980.

[5] G. K. Karapetian, H. J. Engels, and R. J. Gretebeck, "Use of heart rate variability to estimate LT and VT," International Journal of Sports Medicine, vol. 29, no. 8, pp. 652-657, 2008.

[6] N. L. Jones and R. E. Ehrsam, "The anaerobic threshold," Exercise and Sport Sciences Reviews, vol. 10, no. 1, pp. 49-83, 1982.

[7] I. Garcia-Tabar and E. M. Gorostiaga, "A "blood relationship" between the overlooked minimum lactate equivalent and maximal lactate steady state in trained runners. Back to the old days?," Frontiers in Physiology, vol. 9, p. 1034, 2018.

[8] I. Llodio, E. M. Gorostiaga, I. Garcia-Tabar, C. Granados, and L. Sánchez-Medina, "Estimation of the maximal lactate steady state in endurance runners," International Journal of Sports Medicine, vol. 37, no. 7, pp. 539-546, 2016.

[9] I. Garcia-Tabar, L. Sánchez-Medina, J. F. Aramendi, M. Ruesta, J. Ibañez, and E. M. Gorostiaga, "Heart rate variability thresholds predict lactate thresholds in professional world-class road cyclists," Journal of Exercise Physiology Online, vol. 16, no. 5, pp. 38-50, 2013.

[10] M. Buchheit, R. Solano, and G. P. Millet, "Heart-rate deflection point and the second heart-rate variability threshold during running exercise in trained boys," Pediatric Exercise Science, vol. 19, no. 2, pp. 192-204, 2007.

[11] E. M. F. Nascimento, M. A. P. D. M. Kiss, T. M. Santos, M. Lambert, and F. O. Pires, "Determination of lactate thresholds in maximal running test by heart rate variability data set," Asian Journal of Sports Medicine, vol. 8, no. 3, Article ID e58480, 2017.

[12] F. Cottin, C. Medigue, P. Lopes, P. M. Lepretre, R. Heubert, and V. Billat, "Ventilatory thresholds assessment from heart rate variability during an incremental exhaustive running test," International Journal of Sports Medicine, vol. 28, no. 4, pp. 287-294, 2007.

[13] F. Cottin, P. M. Lepretre, P. Lopes, Y. Papelier, C. Medigue, and V. Billat, "Assessment of ventilatory thresholds from heart rate variability in welltrained subjects during cycling," International Journal of Sports Medicine, vol. 27, no. 12, pp. 959-967, 2006.

[14] C. Liu, C. Liu, P Shao et al., "Comparison of different threshold values $\mathrm{r}$ for approximate entropy: application to investigate the heart rate variability between heart failure and healthy control groups," Physiological Measurement, vol. 32, no. 2, pp. 167-180, 2011.

[15] F. Novelli, J. de Araújo, G. Tolazzi, G. Tricot, G. Arsa, and L. Cambri, "Reproducibility of heart rate variability threshold in untrained individuals," International Journal of Sports Medicine, vol. 40, no. 2, pp. 95-99, 2018.

[16] J. Cassirame, N. Tordi, N. Fabre, S. Duc, F. Durand, and L. Mourot, "Heart rate variability to assess ventilatory thresholds in ski-mountaineering," European Journal of Sport Science, vol. 15, no. 7, pp. 615-622, 2015.

[17] L. Mourot, N. Fabre, A. Savoldelli, and F. Schena, "Second ventilatory threshold from heart rate variability: valid when the upper body is involved?," International Journal of Sports Physiology and Performance, vol. 9, no. 4, pp. 695-701, 2014.

[18] R. Di Michele, G. Gatta, A. Di Leo et al., "Estimation of the anaerobic threshold from heart rate variability in an incremental swimming test," Journal of Strength and Conditioning Association, vol. 26, no. 11, pp. 3059-3066, 2012.

[19] S. Quinart, L. Mourot, V. Negre et al., "Ventilatory thresholds determined from HRV: comparison of 2 methods in obese adolescents," International Journal of Sports Medicine, vol. 35, no. 3, pp. 203-208, 2014.

[20] M. M. Sales, C. S. Campbell, P. K Morais et al., "Noninvasive method to estimate anaerobic threshold in individuals with type 2 diabetes," Diabetology \& Metabolic Syndrome, vol. 3, no. 1, pp. 1-8, 2011.

[21] L. Mourot, M. Bouhaddi, S. Perrey, J. D. Rouillon, and J. Regnard, "Quantitative Poincare plot analysis of heart rate variability: effect of endurance training," European Journal of Applied Physiology, vol. 91, no. 1, pp. 79-87, 2003.

[22] B. Casadei, S. Cochrane, J. Johnston, J. Conway, and P. Sleight, "Pitfalls in the interpretation of spectral analysis of the heart rate variability during exercise in humans," Acta Physiologica Scandinavica, vol. 153, no. 2, pp. 125-131, 1995.

[23] N. Candido, N. M. Okuno, C. C. da Silva, F. A. Machado, and F. Y. Nakamura, "Reliability of the heart rate variability threshold using visual inspection and Dmax methods," International Journal of Sports Medicine, vol. 36, no. 13, pp. 1076-1080, 2015.

[24] F. Conconi, M. Ferrari, P. G. Ziglio, P. Droghetti, and L. Codeca, "Determination of the anaerobic threshold by a noninvasive field test in runners," Journal of Applied Physiology, vol. 52, no. 4, pp. 869-873, 1982.

[25] B. Cheng, H. Kuipers, A. C. Snyder, H. A. Keizer, A. Jeukendrup, and M. Hesselink, "A new approach for the determination of ventilatory and lactate thresholds," International Journal of Sports Medicine, vol. 13, no. 7, pp. 518-522, 1992.

[26] R. H. Morton, S. R. Stannard, and B. Kay, "Low reproducibility of many lactate markers during incremental cycle exercise," British Journal of Sports Medicine, vol. 46, no. 1, pp. 64-69, 2012.

[27] D. Harriss and G. Atkinson, "Ethical standards in sport and exercise science research: 2016 update," International Journal of Sports Medicine, vol. 36, no. 14, pp. 1121-2112, 2015.

[28] H. Heck, A. Mader, G. Hess, S. Mucke, R. Muller, and W. Hollmann, "Justification of the 4-mmol/l lactate threshold," International Journal of Sports Medicine, vol. 6, no. 3, pp. 117-130, 1985.

[29] H. H. Dickhuth, L. Yin, A. Niess et al., "Ventilatory, lactate derived and catecholamine thresholds during incremental treadmill running: relationship and reproducibility," International Journal of Sports Medicine, vol. 20, no. 2, pp. 122-127, 1999.

[30] K. M. Souza, T. Grossl, R. J. Babel Junior, R. D. de Lucas, V. P. Costa, and L. G. A. Guglielmo, "Maximal lactate steady state estimated by different methods of anaerobic threshold," 
Revista Brasileira de Cineantropometria e Desempenho Humano, vol. 14, no. 3, pp. 264-275, 2012.

[31] W. G. Hopkins, "Spreadsheets for analisys of validity and reliability," Sportscience, vol. 19, pp. 36-42, 2015.

[32] W. G. Hopkins, S. W. Marshall, A. M. Batterham, and J. Hanin, "Progressive statistics for studies in sports medicine and exercise science," Medicine \& Science in Sports \& Exercise, vol. 41, no. 1, pp. 3-13, 2009.

[33] J. Cohen, Statiscal Power Analysis for the Behavioral Sciences, Lawrance Erlbaum, Mahwah, NJ, USA, 1986.

[34] J. M. Bland and D. G. Altman, "Statistical methods for assessing agreement between two methods of clinical measurement," The Lancet, vol. 327, no. 8476, pp. 307-310, 1986.

[35] I. E. Klein, J. B. White, and S. R. Rana, "Comparison of physiological variables between the elliptical bicycle and run training in experienced runners," Journal of Strength and Conditioning Research, vol. 30, no. 11, pp. 2998-3006, 2016.

[36] G. P. Millet, V. E. Vleck, and D. J. Bentley, "Physiological differences between cycling and running: lessons from triathletes," Sports Medicine, vol. 39, no. 3, pp. 179-206, 2009.

[37] F. Cottin, C. Medigue, P. M. Lepretre, Y. Papelier, J. P. Koralsztein, and V. Billat, "Heart rate variability during exercise performed below and above ventilatory threshold," Medicine \& Science in Sports \& Exercise, vol. 36, no. 4, pp. 594-600, 2004

[38] S. Laborde, E. Mosley, and J. F. Thayer, "Heart rate variability and cardiac vagal tone in psychophysiological research-recommendations for experiment planning, data analysis, and data reporting physiol," Frontiers in Psychology, vol. 8, p. 213, 2017.

[39] T. E. Brown, L. A. Beightol, J. Koh, and D. L. Eckberg, "Important influence of respiration on human R-R interval power spectra is largely ignored," Journal of Applied Physiology, vol. 75, no. 5, pp. 2310-2317, 1993.

[40] S. Chalmers, A. Esterman, R. Eston, and K. Norton, "Standardization of the Dmax method for calculating the second lactate threshold," International Journal of Sports Physiology and Performance, vol. 10, no. 7, pp. 921-926, 2015.

[41] G. R. H. Sandercock and D. A. Brodie, "The use of heart rate variability measures to assess autonomic control during exercise," Scandinavian Journal of Medicine and Science in Sports, vol. 16, no. 5, pp. 302-313, 2006.

[42] J. A. A. C. Heuberger, P. Gal, F. E. Stuurman, W. A. S. de Muinck Keizer, Y. Mejia Miranda, and A. F. Cohen, "Repeatability and predictive value of lactate threshold concepts in endurance sports," PLoS One, vol. 13, no. 11, Article ID e0206846, 2018. 


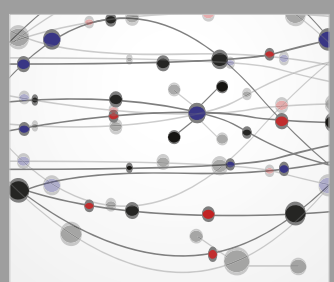

The Scientific World Journal
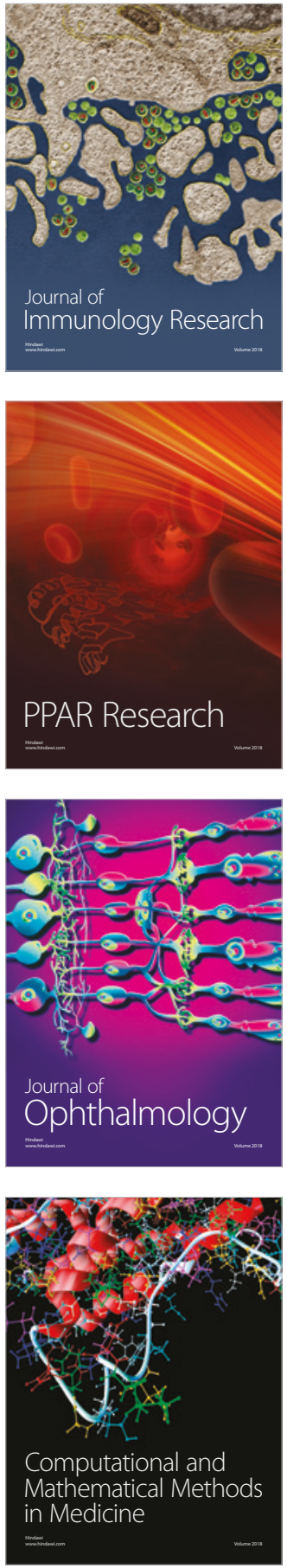

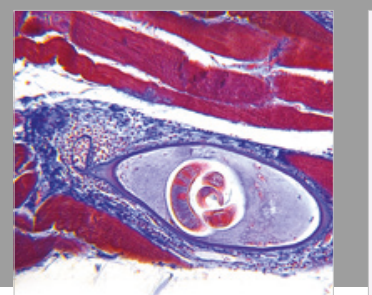

Gastroenterology Research and Practice

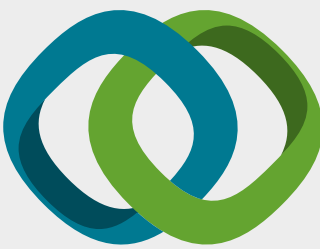

\section{Hindawi}

Submit your manuscripts at

www.hindawi.com
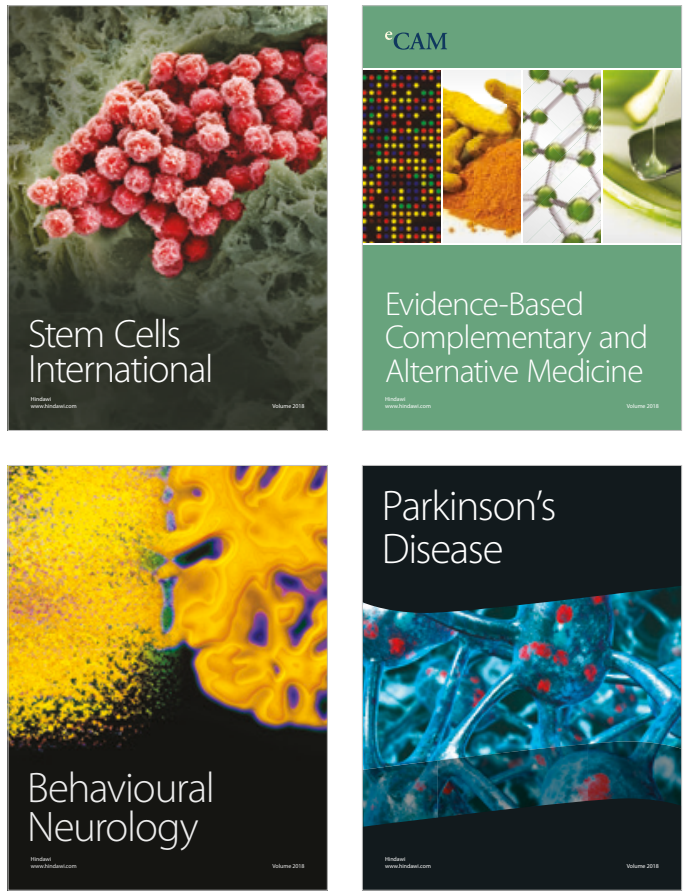

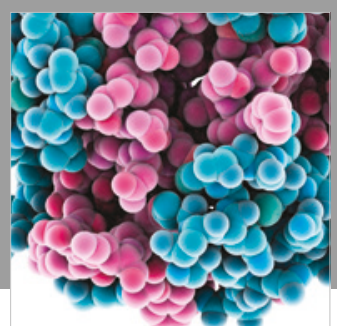

ournal of

Diabetes Research

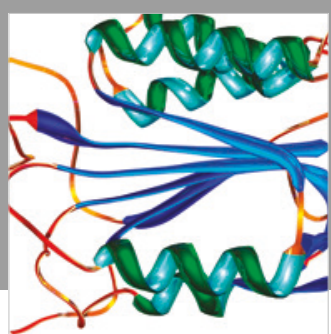

Disease Markers
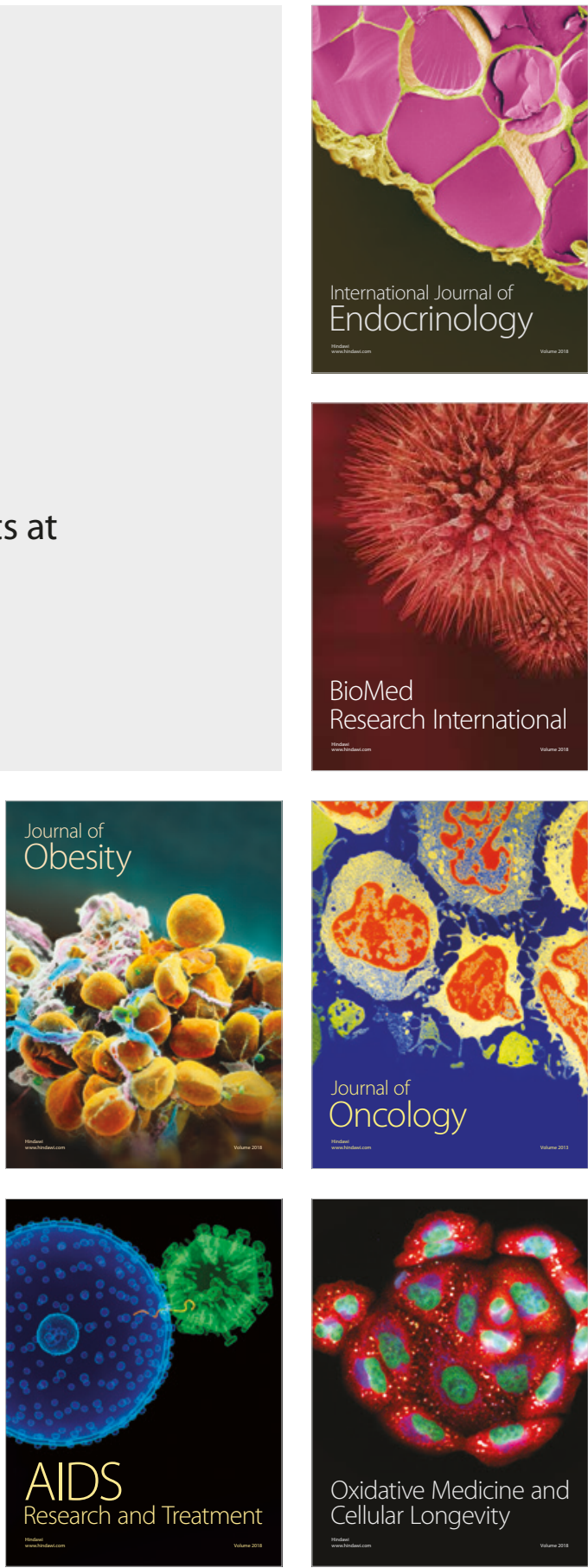